\title{
Search for positively charged strangelets and other related results with E864 at the AGS
}

\author{
Zhangbu Xu† for the E864 Collaboration $\ddagger$ \\ $\dagger$ Physics Department, Yale University, New Haven, CT 06520, USA \\ $\ddagger$ Univ Bari-BNL-UCLA-UC Riverside-Iowa State-Univ Mass-MIT-Penn State- \\ Purdue-Vanderbilt-Wayne State-Yale
}

\begin{abstract}
We report on the latest results in the search for positively charged strangelets from E864's 96/97 run at the AGS with sensitivity of about $8 \times 10^{-9}$ per central collision. This contribution also contains new results of a search for highly charged strangelets with $Z=+3$. Production of light nuclei, such as ${ }^{6} \mathrm{He}$ and ${ }^{6} \mathrm{Li}$, is presented as well. Measurements of yields of these rarely produced isotopes near midrapidity will help constrain the production levels of strangelets via coalescence. E864 also measures antiproton production which includes decays from antihyperons. Comparisons with antiproton yields measured by E878 as a function of centrality indicate a large antihyperon-to-antiproton ratio in central collisions.
\end{abstract}

\section{Introduction}

Strangelets are small color-singlet hadrons with baryon number $A>1$ which contain about equal numbers of $\mathrm{u}, \mathrm{d}$ and s quarks. Many of the theoretical calculations 11, 2, 3] based on the phenomological bag model suggest that Strange Quark Matter (SQM) might be metastable or even absolutely stable. Ultimately only experiments can prove their existence or nonexistence.

Relativistic Heavy Ion Collisions create hot and dense nuclear (or quark) matter, which offer a unique opportunity to search for strangelets in accelerator facilities $[$. The experimental signature of strangelets used to date is their low charge-to-mass ratio. This is based on the fact that strangelets have one more quark flavor with negative charge $\left(-\frac{1}{3}\right)$ than the normal 2-flavor nuclear matters. Recent investigations [1] suggest that strangelets produced in heavy ion collisions might even be highly negatively charged.

There are three classes of strangelet production models in Heavy Ion Collisions: distillation of Quark-Gluon Plasma (QGP) scenario 8, 9], coalescence model 10], and thermal model[14]. The QGP distillation scenario assumes that the strangelet formation is a two-step process. It is creation of QGP followed by QGP decay into strangelet. These two processes are in the speculative stage 11. Some estimations are available in reference [8, 13] in which the QGP is assumed to break up into small droplets before distillation. Coalescence models calculate the production of hyperfragments. The hyperfragments are formed from individual nucleons and they subsequently decay to strangelets provided that strangelets are more stable. A thermal model 14 need not discuss specific reaction mechanisms, since its major ingredients are thermal and chemical equilibrium. 
To prove the existence of strangelet one needs to find the strangelet. But to prove the nonexistence of strangelet, we need to know the production mechanism well. Unfortunately, even the formation of QGP is yet to be seen. The measurement of antihyperon-to-antiproton ratio will help us understand the system better even within the framework of hot and dense nuclear matter while large antihyperon-to-antiproton ratio 15 is consistent with QGP formation. Measurements of production of light nuclei are keys to understanding the possible strangelet production via coalescence 10 and they also provide other important information about the colliding system 27.

This contribution presents recent results in the search for positively charged strangelet with E864 and discusses the significance of the results and their implications with the help of light nuclei results. The antihyperon-to-antiproton ratios or $\left(\bar{\Lambda}+\bar{\Sigma}^{0}+1.1 \bar{\Sigma}^{+}\right) / \bar{p}$ vs. centrality implied from $\bar{p}$ measurements by E864 and E878 are presented as well.

\section{E864 apparatus}

E864 is an open geometry, high data rate spectrometer designed to search for strangelets[5, 6, 7] and measure the production of many particle species in high energy nucleus-nucleus collisions at the AGS. We can have two relatively independent mass measurements with the E864 apparatus(Fig 11) : the tracking system and the hadronic calorimeter. They identify particles and reject background powerfully with the confirmation of each other. The tracking system has two dipole analyzing magnets (M1 and M2) followed by three hodoscope planes(H1, H2 and H3). There are two straw stations (S2 and S3), each with three close-packed double planes. Each scintillating hodoscope plane has 206 vertical slats, and there are more than 1000 straw tubes in each straw plane. The tracking system measures momentum, charge and velocity $(\beta)$ of charged particles with a mass resolution of about $3 \%$ in the region of interest. Charge misidentification is less than 1 in 10 billion due to three redundant charge measuremnts. There is an additional mass measurement from hadronic calorimeter 16 which has good energy $(\Delta E / \sqrt{E}=0.344 / \sqrt{E}+0.035)$ and time $\left(\sigma_{t} \simeq 400 p s\right)$ resolutions. It is made of 754 towers of scintillating fibre embedded lead. A vacuum tank is along the beam line to reduce the background from beam particles interacting with air. The total length of the apparatus is about 28 meters. The incident beam is $11.5 \mathrm{GeV} / \mathrm{c} \mathrm{Au}$ beam on Pt target with $60 \%$ interaction length. The spectrometer fields of M1 and M2 are set to their highest field $+1.5 \mathrm{~T}$ to reduce background (sweep out high $Z / A$ particles) and achieve best tracking resolution for positively charged strangelets.

The trigger consists of good beam definition, a multiplicity requirement 17 and a level II high mass trigger 18. Only events of the $10 \%$ most central collisions are collected since strangelets are most likely to be produced in central collisions. High sensitivity and open geometry are keys of E864. We achieve the high sensitivity by a high-mass level II trigger - Late-Energy Trigger (LET). There is a two-dimensional (Energy and TOF) programmable lookup table for every calorimeter channel to setup for different topics. The LET rejects those events without any high mass candidate and achieve a rejection factor of about 70 while maintaining good efficiency $(>85 \%)$ for high masses. There are about 200 million LET events or 13 billion $10 \%$ most central events sampled in the whole data set for positively charged or neutral strangelet searches.

Offline cuts are used to further refine the candidate selection. For any particle, tracking mass and calorimeter mass have to be consistent with each other. These 
confirmations are performed by energy and TOF consistency cuts. A upper limit cut on particle velocity $(\beta)$ is used to maintain good mass resolution and clean up the background since $\sigma_{m} / m$ scales with $\gamma^{2}$. Tracking $\chi^{2}$ and shower quality cuts are studied extensively.

\section{Results}

\subsection{Strangelet searches}

We have conducted the full analysis of $1996 / 97$ 's ' $+1.5 \mathrm{~T}$ ' data set for strangelets with charge $=+1,+2,+3$.

There are two classes of background which we have to deal with. The background in the tracking system is the result from multiple scattering and neutron-proton charge exchange interactions. Fluctuations of energy measurements and overlapping showers are background in the calorimeter measurements. These two background sources are relatively independent. Simulations showed that we are able to achieve a rejection level of $<10^{-10}$ per central interaction[20].

With all the cuts we used in analysis of data from the previous run [6, 21, 22], we observe 3 candidates with mass between 5 and $10 \mathrm{Gev} / \mathrm{c}^{2}$. But when we check for additional evidence of double interactions using the calorimeter, we conclude that the 3 candidates are probably normal particles scattered and their showers in calorimeter are contaminated by a second interaction 19, 23. The efficiency of this additional cut is about $85 \%$. In fig 2, the mass measured from the tracking system of charge $=+2$ is plotted. It is clear that consistency cuts between tracking and calorimeter measurements and tighter $\beta$ cut do clean up the spectrum. We clearly see a ${ }^{6} \mathrm{He}$ peak but we do not see any ${ }^{8} \mathrm{He}$ or exotic particles. We conclude that there is no candidate with $1.0<y<2.1$ and $m>7 G e V / c^{2}$. We extend our analysis to include $|Z| \geq 3$ particles and indeed we are able to see a clear ${ }^{6} \mathrm{Li}$ peak. With a loose $\beta<0.985$ cut, no particle with $m>8 \mathrm{GeV} / \mathrm{c}^{2}$ is found. However, when the limits are calculated, a tighter cut $(\beta<0.972)$ is used. An analysis with the looser cut is being carried out and should give slightly better limits.

Because of the finite acceptance, we choose the following production model to calculate the sensitivities and limits :

$$
\frac{d N}{d y d p_{t}} \propto p_{t} \exp \left[-\frac{2 p_{t}}{<p_{t}>}\right] \exp \left[-\frac{\left(y-y_{c m}\right)^{2}}{2 \sigma_{y}^{2}}\right]
$$

where $\left\langle p_{t}\right\rangle=0.6 \sqrt{A} G e V / c$ is the mean transverse momentum, $y_{c m}=1.6$ is the center-of-mass rapidity and $\sigma_{y}=0.5$ is the standard width of the rapidity distribution of the produced strangelet. It is worth pointing out that because of the large rapidity and transverse momentum coverage, the results depend weakly on the model chosen. E864 is capable of detecting weak-decay strangelets with lifetime of about 50ns or greater.

We compute the limits at $90 \%$ Confidence Level (C.L.) for strangelets of $\mathrm{Z}=+1$, +2 and +3 with mass between about 10 to $50 \mathrm{Gev} / \mathrm{c}^{2}$. These results represent the best limits at AGS energies 团. Fig 3 shows the preliminary limits together with our previous results [6] and predictions from coalescence, QGP distillation and thermal model. Interpretations and implications of the results will be discussed in next section. 


\subsection{Light nuclei}

In addition to searching for positively charged strangelet, we measure the production of light nuclei near midrapidity up to $A=6$ in this data set. Fig 2 shows preliminary measurements of the yields of ${ }^{6} \mathrm{He}\left(J^{P}=0^{+}\right)$and ${ }^{6} \mathrm{Li}\left(J^{P}=1^{+}\right)$. Together with the analyses of other E864 data sets 24, 25], we observe an exponential decrease of the yields of particle production as a function of nuclear number A near midrapidity (center of mass of the system). That is

$$
\left.\frac{d N_{A}}{d y d p_{t}}\right|_{p_{t} \simeq 0, y \simeq 1.9} \propto(1 / 50)^{A}
$$

This means that the penalty factor of adding a nucleon to a nuclear cluster is about 50 for all nuclei up to $A=6$ near midrapidity at $p_{t} \simeq 0$. From this observation, one can make a naive model of the particle production, namely:

$$
N_{A} \simeq 157 \times\left(\frac{1}{50}\right)^{A-1} \times \lambda_{s}{ }^{|S|}
$$

where 157 is the total number of initial protons, $\lambda_{s}$ is the strangeness penalty factor [10, 26] and $|S|$ is the total strangeness in the 'cluster'. This naive model assumes complete stopping and does not take into account the change of the spectrum with mass and the spin factor. But it describes the trend of the production level.

\section{3. $\bar{p}$ production vs. centrality}

E864 has previously measured $\bar{p}$ production in 10\% most central collisions 15, 28, 22]. Additional mininum bias data were taken in the 1996/97 run with LET trigger. When the measured $\bar{p}$ production shown in Fig 4 is compared with that from E878 29, 15] in different centralities, we see a strong centrality dependence of the level of disagreement between the two experiment measurements. This can be explained by the enhancement of antihyperon production in central collisions, because E864 accepts all the $\bar{p}$ 's from antihyperon $\left(\bar{\Lambda}, \bar{\Sigma}^{0}, \overline{\Sigma^{+}}\right)$decays while E878 accepts only a small fraction of them . Detail comparisons can be found in 15, 28, 22]. From the difference of the acceptance and difference of the detected $\vec{p}$ 's, we compute the antihyperon-to-antiproton ratios $\left(\bar{\Lambda}+\bar{\Sigma}^{0}+1.1 \bar{\Sigma}^{+}\right) / \bar{p}$ in each centrality bin, where acceptance and decay branching ratios are properly taken into account. In Fig 1 , we plot the low edge of the ratios at $98 \%$ C.L. together with the most probable values of the ratios as a function of centrality. It reaches the most probable value of 3.5 and is higher than 2.3 at $98 \%$ C.L. in central collisions. The interpretation is quite consistent since the ratio in peripheral collisions is consistent with that of $\mathrm{p}+\mathrm{p}$ collisions at similar energy. We are looking forward to the forthcoming direct $\bar{\Lambda}$ measurements [30] at the AGS.

\section{Interpretation of Strangelet Limits}

\subsection{Coalescence and thermal models}

Coalescence 10 and thermal 14 models predict the production of hyperfragments. It is noticed that [10] [14] overpredict the production of light nuclei 24, 25] and therefore we expect overpredictions of hyperfragment/strangelet production from these models.

From Equation (3) and our most probable sensitivity at low mass range $(1.4 \times$ $10^{-8} / 2.3$ ), we can translate our limits per central collision to limits in baryon number 
and strangeness content $[7]$ :

$$
A+(0.41 \times|S|)<7.1
$$

where $\lambda_{s}=0.2$ 26. For any combination of $\mathrm{A}$ and $\mathrm{S}$ satisfying Equation (4), we have the sensitivity for strangelets produced by coalescence if they exist.

Another important message from Equation (3) is that it is very unlikely to produce a large strangelet in a normal medium. For example, production of an $A=10$ and $|S|=0$ object will be at the level of $8 \times 10^{-14}$ per central collision. On the other hand, this implies that positively charged strangelets can be a possible clean signature of QGP formation [9] which will not be confused by normal nuclei or strangelets produced via 'coalescence'.

\subsection{Distillation of $Q G P$}

Our limits largely rule out the predictions from [8] and are at the same order of or below predictions from [13] for $m \sim 10$. However, predictions listed from [13] are from their early predictions in which a QGP is assumed to happen in every central collision at the AGS energy and an optimistic mass formula is used. Further improvement in

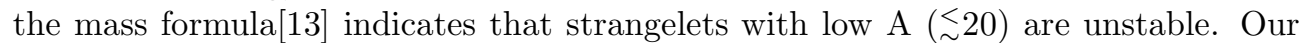
limits are not sensitive enough to challenge the region where one might expect stability according to this model.

Our limits do constrain the sequence of QGP production followed by QGP distillation into strangelet in a model independent way[6]. For large strangelets which are predicted to be more stable, our data restrict these processes at the $90 \%$ confidence level as follows:

$$
\mathrm{B}(\mathrm{Au}+\mathrm{Pt} \rightarrow \mathrm{QGP}) \times \mathrm{B}(\mathrm{QGP} \rightarrow \text { Strangelet }) \stackrel{<}{\sim} \times 10^{-9},
$$

\section{Conclusions and future prospects}

In summary, we have found no evidence of positively charged strangelets produced in $11.5 \mathrm{GeV} / \mathrm{c}$ per nucleon $\mathrm{Au}+\mathrm{Pt}$ collision and set a $90 \%$ confidence level upper limit of about $8 \times 10^{-9}$ per $10 \%$ most central collision for $|Z|=+1,+2,+3$ strangelets over a wide mass range and with proper lifetimes of $>50 \mathrm{~ns}$. This represents the best sensitivity at the AGS energies. We have measured production of $A=6$ nuclei near midrapidity and found that the penalty factor of adding a nucleon to a cluster stays at about 50. Comparisons of $\bar{p}$ measurements between E864 and E878 as a function of centrality suggest an enhanced antihyperon-to-antiproton ratio in central collisions.

In the near future, we will combine two data sets ('+1.5T' and '-.75T') together to improve our sensitivities.

The strangeness penalty factor for adding hyperon to a nuclear cluster has never been measured in high energy heavy ion collisions. We have collected about 250 million LET events ( 12 billion central events sampled). The unique high sensitivity and open geometry give us opportunity to measuring the production of ${ }_{\Lambda}^{3} H$ and ${ }_{\Lambda}^{4} H$ through their two-body mesonic decay channel[31]. There are also data available for studying nuclear resonant states, such as ${ }^{5} \mathrm{Li},{ }^{5} \mathrm{He}$. With these new studies, we hope to better understand the dynamics of the high energy heavy ion collisions and provide more information for future experiments in searching for strangelets via coalescence. 


\section{Acknowledgments}

We gratefully acknowledge the excellent support of the AGS staff. This work was supported by grants from the U.S. Department of Energy's Hight Energy and Nuclear Physics Divisions, the U.S. National Science Foundation and the Istituto di Fisica Nucleara of Italy.

\section{Reference}

[1] Schaffner-Bielich J et al 1997 J. Phys. G: Nucl. Part. Phys. 23 p 2107

[2] Chin S A and Kerman A K 1979 Phys. Rev. Lett. 43 p 1292

[3] Madsen J 1995 Strangeness in Hadronic Matter (S '95) ed J Rafelski (New York: AIP) p 32

[4] Sandweiss J These proceedings

[5] Barish K N 1997 J. Phys. G: Nucl. Part. Phys. 23 p 2127

[6] Armstrong T A et al 1997 Phys. Rev. Lett. 79 p 3612

[7] Armstrong T A et al 1997 Nucl. Phys. A625 p 494

[8] Liu H and Shaw G L 1984 Phys. Rev. D30 p1137

[9] Greiner C et al 1987 Phys. Rev. Lett. 58 p 1825

[10] Baltz A et al 1994 Phys. Lett. B325 p 7

[11] Greiner C These proceedings

[12] Kapusta J et al 1995 Phys. Rev. C52 p 2725

[13] Crawford H et al 1992 Phys. Rev. D45 p $857 — 1993$ Phys. Rev. D48 p 4474

[14] Braun-Munzinger P and Stachel J 1995 J. Phys. G: Nucl. Part. Phys. p L17

[15] Armstrong T A et al 1997 Phys. Rev. Lett. 79 p 3351 (references therein)

[16] Armstrong T A et al 1998 Nucl. Instrum. Methods A 406 p 227

[17] Haridas $\mathrm{P}$ et al 1997 Nucl. Instrum. Methods A 385 p 412

[18] Hill J et al Nucl. Instrum. Methods $A$ (to be published)

[19] Xu Z PhD Thesis, Yale University, in preparation

[20] Sandweiss J et al 1991 E864 proposal: Measurements of Rare Composite Objects and High Sensitivity Searches for Novel Forms of Matter Produced in High Energy Heavy Ion Collisions

[21] Coe S D 1997 PhD Thesis, Yale University

[22] Nagle J L 1997 PhD Thesis, Yale University

[23] Munhoz M 1998 These proceedings; Van Buren G 1998 These proceedings

[24] Pope J K 1996 Proceedings of Heavy Ion Physics at the AGS (HIPAGS) ed C A Pruneau et al WSU-NP-96-16 p $119-1997$ PhD Thesis, Yale University

[25] George N 1998 PhD Thesis, Yale University, in preparation

[26] Dover C 1995 Nucl. Phys. A 590 p 333

[27] Heinz U 1998 These proceedings

[28] Lajoie J G 1997 PhD Thesis, Yale University

[29] Beavis D et al 1995 Phys. Rev. Lett. 75 p 3078

[30] Ogilvie C 1998 These proceedings

[31] Xu Z et al Observability of ${ }_{\Lambda}^{4} H$ with E864, E864 private document 


\section{Figure captions}

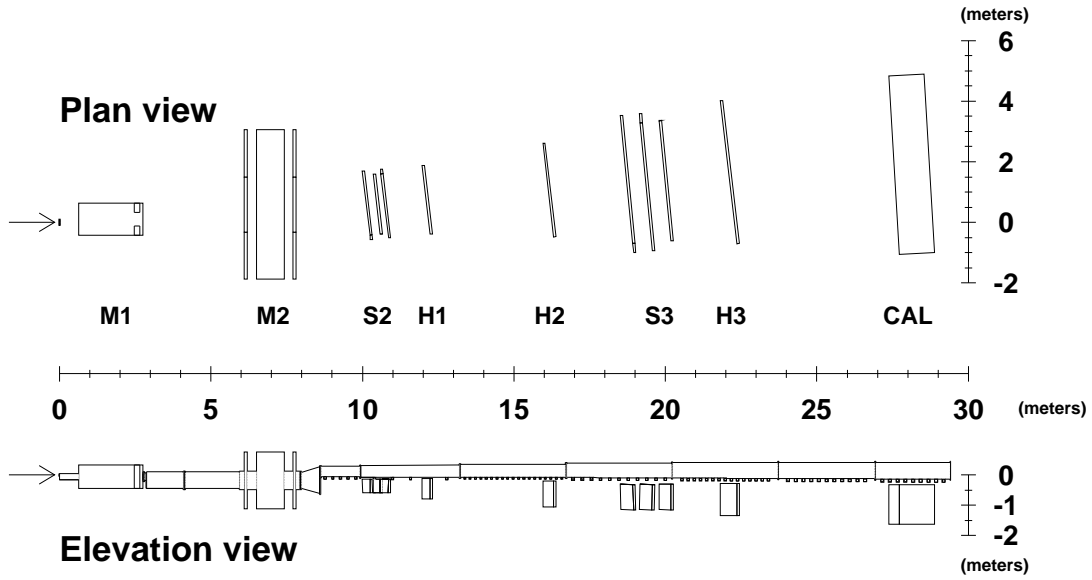

Figure 1. Schematic views of the E864 spectrometer. In the plan view, the downstream vacuum chamber is not shown. M1 and M2 are dipole analyzing magnets, S2 and S3 are straw tube arrays. H1, H2 and H3 are scintillator hodoscopes, and CAL is a hadronic calorimeter. Scales are in meters.

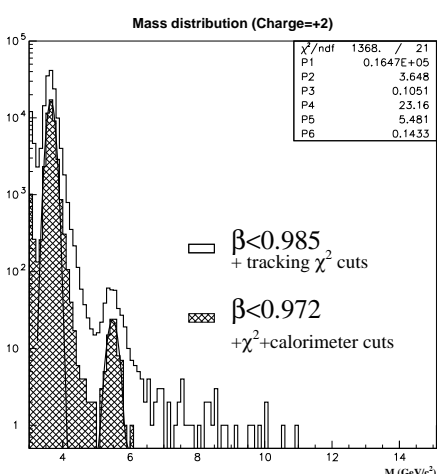

(a) $\mathrm{Q}=+2$ strangelet searches

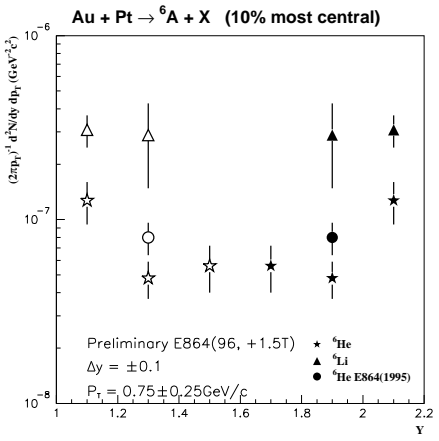

(b) ${ }^{6} \mathrm{He}$ and ${ }^{6} \mathrm{Li}$ invariant yeilds

Figure 2. (a) $Z=+2$ spectrum of tracking mass with various cuts. Tracking $\chi^{2}$ cuts, calorimeter cuts and tight $\beta$ cut are applied in sequence. (b) The measured ${ }^{6} \mathrm{Li}$ and ${ }^{6} \mathrm{He}$ yields vs. rapidity at $p_{t} \simeq 0$. Open symbols are reflections of the filled ones. Filled circle is measurement from previous run with less statistics, therefore, larger rapidity and $p_{t}$ bin. 


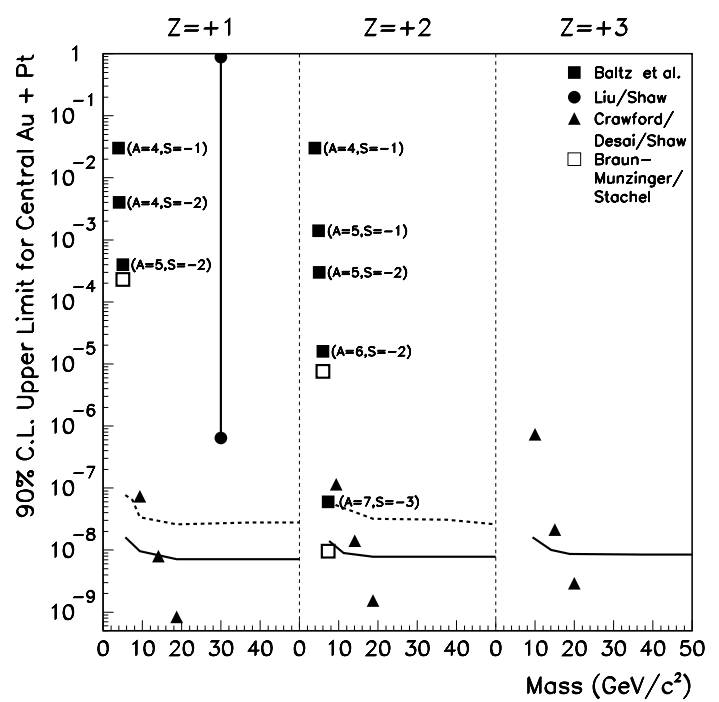

Figure 3. $90 \%$ C.L. limits in $10 \%$ central collisions for $\mathrm{Z}=+1,+2$ and +3 strangelet production with lifetimes of about 50ns or greater. Solid lines are preliminary limits from $1996 / 97^{\prime}$ s run, while the dashed lines are those from $1995^{\prime}$ s run. The full squares are prediction based on coalescence from [10]. The open squares are predictions based on thermal model from [14]. The full circles with the line are the range of predictions based on QGP formation from [8]. The full triangles are predictions based on QGP formation from [13] at $14.5 \mathrm{GeV} / \mathrm{c}$ beam energy. (See text for detail.)

$\mathrm{E} 864 \mathrm{Au}+\mathrm{Pb} \rightarrow \mathrm{pbar}+\mathrm{X}$ at $11.5 \mathrm{~A} \mathrm{GeV} / \mathrm{c}$

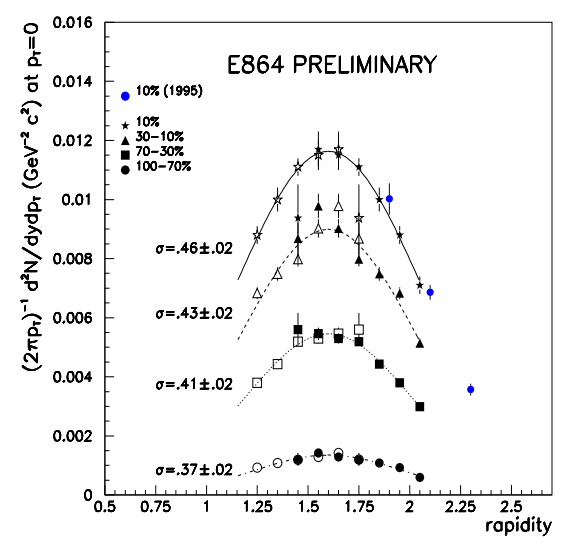

(a) $\bar{p}$ centrality

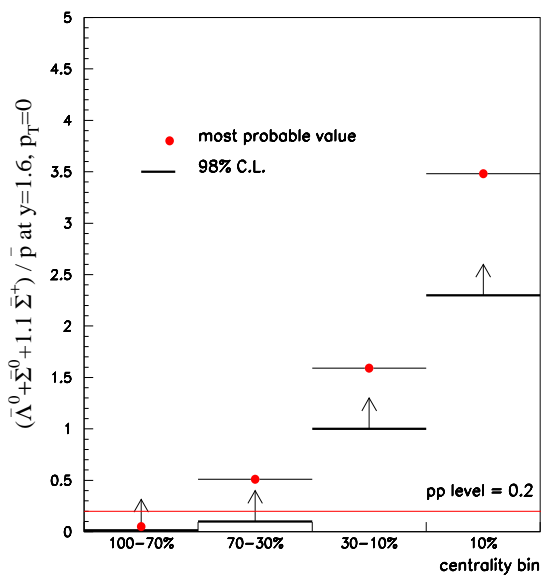

(b) $\bar{Y} / \bar{p}$

Figure 4. (a) $\bar{p}$ measurements in 4 different centrality bins. $\sigma$ is the width of gaussian fit. (b) $\left(\bar{\Lambda}+\bar{\Sigma}^{0}+1.1 \bar{\Sigma}^{+}\right) / \bar{p}$ and its low edge at $98 \%$ C.L. as a function of centrality from the difference of $\bar{p}$ measurements by E864 and E878. 